\title{
ON THE MAXIMAL POLAR QUOTIENT OF AN ANALYTIC PLANE CURVE
}

\author{
Arkadiusz Ploski*
}

\begin{abstract}
We give an explicit formula for the maximal polar quotient of a plane curve singularity with some applications to the Lojasiewicz exponent and the $C^{0}$-degree of sufficiency.
\end{abstract}

\section{Introduction}

Let $C\{X, Y\}$ be the ring of convergent power series in two variables $X, Y$. If $f, \phi \in C\{X, Y\}$, we denote by $(f, \phi)_{0}$ the intersection multiplicity of $f$ and $\phi$, equal to the $\boldsymbol{C}$-codimension of the ideal $(f, \phi)$ generated by $f$ and $\phi$ in $C\{X, Y\}$. We use ord $f$ to denote the order of the series $f$. If $f, \phi$ are without constant term, then $(f, \phi)_{0} \geq($ ord $f)$ (ord $\left.\phi\right)$, the equality holding if and only if $f, \phi$ are transverse. Let $t=t(X, Y) \in C\{X, Y\}$ be a regular parameter i.e. a series of order 1. Assume that $f \in \boldsymbol{C}\{X, Y\}$ is a reduced (i.e. without multiple factors) power series and $t$ does not divide $f$. The rational numbers $(f, \phi)_{0} /(t, \phi)_{0}$, where $\phi$ runs over irreducible factors of the Jacobian $J=\partial(t, f) / \partial(X, Y)$, are called polar quotients of $f$ with respect to $t$. If $t=b X-a Y$ then we speak about polar quotients with respect to the direction $(a: b) \in \boldsymbol{P}^{1}(\boldsymbol{C})$. Clearly the set of polar quotients is finite. It is empty if and only if $(\partial(t, f) / \partial(X, Y))(0,0) \neq 0$. If $t$ and $f$ are transverse, then the polar quotients are of the form $(f, \phi)_{0} /$ ord $\phi$ and are also called polar invariants. They are topological invariants of the singularity $f=0$ (see [T], [LMW]). If $t$ and $f$ are not transverse the notion of polar quotient is also interesting, especially in the case of the singularities at infinity of plane algebraic curves (see $[\mathrm{Eph}]$ and [Lê]). Casas-Alvero ([C-A], Chapter 6, Theorem 6.11.5) calculated polar quotients in the general case of reduced power series using the infinitely near points. However, the formulas he got are not so explicit as that given by Merle $([\mathrm{M}]$, Théorème 3.1) in the case of one branch. In this note we study the maximal polar quotient $q_{0}(f, t)$ of $f$ with respect to $t$ :

1991 Mathematics Subject Classification: 32S05

Key words and phrases: plane curve singularity, polar quotients, Lojasiewicz exponent, degree of sufficiency.

* Supported in part by the KBN grant No 2 P03 A 00115.

Received May 22, 2000; revised August 28, 2000. 
$q_{0}(f, t)=\sup \{q \in \boldsymbol{R}: q$ is a polar quotient of $f$ with respect to $t\}$. If $(\partial(t, f) / \partial(X, Y))(0,0) \neq 0$ then $q_{0}(f, t)=-\infty$ by convention.

Our aim is to give an explicit formula for $q_{0}(f, t)$ (Theorem 1.3 of this paper) by means of the maximal polar quotients of the branches and some intersection multiplicities. Our result is inspired by Kuo and Lu's paper [KL], to prove it we use the Kuo-Lu lemma that enables us to locate the Puiseux roots of $\partial f / \partial Y=0$ relatively to the roots of $f=0$ ([KL], Lemma 3.3). As an application of our formula we show that for a given $f$ the $\sup \left\{q_{0}(f, t): t\right.$ does not divide $f\}$ is attained if $f$ and $t$ are transverse (Corollary 1.4). The maximal polar quotient are of particular interest. Teissier in his fundamental paper $[\mathrm{T}]$ proved (in the case of isolated hypersurface singularities) that the Lojasiewicz exponent $\mathscr{L}_{0}(f)$ and the $C^{0}$-degree of sufficiency are determined by the maximal polar quotient with respect to the generic direction. Using Teissier's result we give formulas for $\mathscr{L}_{0}(f)$ and $\operatorname{Suff}_{0}(f)$ (Corollary 1.5) and a correct version (Corollary 1.8) of a formula for $\operatorname{Suff}_{0}(f)$ proposed by Lichtin in [Li]. In the appendix we reprove the main result of $[\mathrm{KL}]$. We give also an example showing that the geometric interpretation of this result given in $[\mathrm{KL}]$ is not exact.

\section{Main result}

Let $f \in C\{X, Y\}$ be an irreducible power series. Recall that the semigroup $\Gamma_{0}(f)$ of $f$ is the set of all intersection numbers $(f, \phi)_{0}$ where $\phi$ runs over all power series $\phi \in \boldsymbol{C}\{X, Y\}$ such that $\phi \notin(f) \boldsymbol{C}\{X, Y\}$. Let $\bar{\beta}_{0}, \ldots, \bar{\beta}_{g}$ be the minimal system of generators of $\Gamma_{0}(f): \bar{\beta}_{0}=\min \left(\Gamma_{0}(f) \backslash\{0\}\right)=\operatorname{ord} f, \bar{\beta}_{i}=$ $\min \left(\Gamma_{0}(f) \backslash\left(\boldsymbol{N} \bar{\beta}_{0}+\cdots+\boldsymbol{N} \bar{\beta}_{i-1}\right)\right)$ for $i=1, \ldots, g, \boldsymbol{N} \bar{\beta}_{0}+\cdots+\boldsymbol{N} \bar{\beta}_{g}=\Gamma_{0}(f)$. Let $\operatorname{GCD}\left(\bar{\beta}_{0}, \ldots, \bar{\beta}_{i}\right)$ stand for the greatest common divisor of $\bar{\beta}_{0}, \ldots, \bar{\beta}_{i}$.

Proposition 1.1. Suppose that $f$ is an irreducible power series with ord $f>$ 1. Then for every regular parameter $t$ we have

$$
q_{0}(f, t)=\frac{\mathrm{GCD}\left(\bar{\beta}_{0}, \ldots, \bar{\beta}_{g-1}\right) \bar{\beta}_{g}}{(t, f)_{0}} .
$$

The proposition above can be easily deduced from the generalization of Merle's result $([\mathrm{M}]$, Théorème 3.1) given by Ephraim ([Eph], Lemma 1.6). We give a direct proof of (1.1) in Section 4 of this paper.

Remark 1.2. If ord $f=$ ord $t=1$ and $(\partial(t, f) / \partial(X, Y))(0,0) \neq 0$ then $q_{0}(f, t)=-\infty$, because the set of polar quotients is empty (by convention $\sup \emptyset=-\infty)$. If ord $f=\operatorname{ord} t=1$ and $(\partial(t, f) / \partial(X, Y))(0,0)=0$ then there is only one polar quotient and it is equal to 1 . Hence $q_{0}(f, t)=1$.

In the sequel we put $\max \{-\infty, a\}=a$ for every $a \in \boldsymbol{R}$. The main result of this note is 
THEOREM 1.3. Let $f=f_{1} \cdots f_{r}(r \geq 2)$, with $f_{i} \in \boldsymbol{C}\{X, Y\}$ irreducible, be a reduced power series. Let $t$ be a regular parameter that does not divide $f$. Then

$$
q_{0}(f, t)=\max _{i=1}^{r}\left\{\max \left\{q_{0}\left(f_{i}, t\right), \max _{j \neq i} \frac{\left(f_{i}, f_{j}\right)_{0}}{\left(t, f_{j}\right)_{0}}\right\}+\frac{1}{\left(t, f_{i}\right)_{0}} \sum_{j \neq i}\left(f_{i}, f_{j}\right)_{0}\right\} .
$$

We give the proof of (1.3) in Section 4. If $f$ and $t$ are transverse then our theorem is an intersection theoretical counterpart of the main theorem of Kuo and $\mathrm{Lu}$ (see $[\mathrm{KL}]$, Theorem A). Eggers in $[\mathrm{E}]$ developed Kuo and Lu's ideas to calculate the polar quotients with respect to the generic direction in terms of characteristics of branches and their intersection multiplicities coded by means of tree-models (see $[\mathrm{Ga}]$ for further developments). In this case our result could be also proved by using Eggers' theorem ([E], Satz 2.1). Let us define for any reduced power series $f$ the invariant $q_{0}(f)$ by putting

(i) if ord $f=1$, then $q_{0}(f)=-\infty$,

(ii) if $f$ is irreducible, ord $f>1$ and $\bar{\beta}_{0}, \bar{\beta}_{1}, \ldots, \bar{\beta}_{g}$ is the minimal system of generators of the semigroup $\Gamma_{0}(f)$ then

$$
q_{0}(f)=\frac{\operatorname{GCD}\left(\bar{\beta}_{0}, \ldots, \bar{\beta}_{g-1}\right) \bar{\beta}_{g}}{\bar{\beta}_{0}},
$$

(iii) if $f=f_{1} \cdots f_{r}, r \geq 2$ with irreducible $f_{i}(i=1, \ldots, r)$ then

$$
q_{0}(f)=\max _{i=1}^{r}\left\{\max \left\{q_{0}\left(f_{i}\right), \max _{j \neq i} \frac{\left(f_{i}, f_{j}\right)_{0}}{\operatorname{ord} f_{j}}\right\}+\frac{1}{\operatorname{ord} f_{i}} \sum_{j \neq i}\left(f_{i}, f_{j}\right)_{0}\right\} .
$$

Using (1.1), (1.2) and (1.3) we get

COROLlaRY 1.4. Suppose ord $f>1$. Then for every regular parameter $t$ that does not divide $f$ we have $q_{0}(f, t) \leq q_{0}(f)$. If $t$ and $f$ are transverse then $q_{0}(f, t)=q_{0}(f)$.

Let $S$ be an analytic set near $0 \in C^{2}$. We put $\mathscr{L}_{0}(f, S)=\inf \{\theta>0$ : $|\operatorname{grad} f(z)| \geq C|z|^{\theta}$ for $z \in S$ near 0$\}$ and call $\mathscr{L}_{0}(f)=\mathscr{L}_{0}\left(f, \boldsymbol{C}^{2}\right)$ the Lojasiewicz exponent of $f$. We say that $\mathscr{L}_{0}(f)$ is attained along $S$ if $\mathscr{L}_{0}(f, S)=\mathscr{L}_{0}(f)$. Let $\operatorname{Suff}_{0}(f)$ be the $C^{0}$-degree of sufficiency i.e. the smallest integer $r$ such that $f$ is topologically equivalent to $f+g$ for all $g$ with ord $g \geq r+1$. A historical note about these two notions is given in [LW].

COROllary 1.5. If $f$ is reduced and ord $f>1$ then $\mathscr{L}_{0}(f)=q_{0}(f)-1$ and $\operatorname{Suff}_{0}(f)=\left[q_{0}(f)\right]$.

Proof. According to $[\mathrm{T}]$ (Sec. 1, Corollaire 2 and Sec. 3, Théorème 8) there is a Zariski-open subset $U \subset \boldsymbol{P}^{1}(\boldsymbol{C})$ such that $\mathscr{L}_{0}(f)=q_{0}(f, b X-a Y)-1$, $\operatorname{Suff}_{0}(f)=\left[q_{0}(f, b X-a Y)\right]$ for $(a: b) \in U$. On the other hand if the line $b X-$ $a Y=0$ is not tangent to the curve $f(X, Y)=0$ then $q_{0}(f, b X-a Y)=q_{0}(f)$ by Corollary 1.4. 
The following result was proved independently by Bogusławska ([B], Theorem 2) and Kuo and Parusiński ([KP], Theorem 3.1).

COROllary $1.6([\mathrm{~B}],[\mathrm{KP}])$. If the line $b X-a Y=0$ is not tangent to the curve $f(X, Y)=0$ then the Eojasiewicz exponent $\mathscr{L}_{0}(f)$ is attained on the polar curve $a(\partial f / \partial X)+b(\partial f / \partial Y)=0$.

Proof. Assume that the line $b X-a Y=0$ is not tangent to the curve $f(X, Y)=0$. Using parametrizations of the branches of the polar curve $a(\partial f / \partial X)+b(\partial f / \partial Y)=0$ we check that $\mathscr{L}_{0}(f,\{a(\partial f / \partial X)+b(\partial f / \partial Y)=0\})=$ $q_{0}(f, b X-a Y)-1$. Then we use (1.4) and (1.5).

Remark 1.7. Let $f=f_{1} \cdots f_{r}(r \geq 2)$ be a reduced power series such that the irreducible factors $f_{i}$ are pairwise transverse that is $\left(f_{i}, f_{j}\right)_{0}=\left(\right.$ ord $\left.f_{i}\right)\left(\right.$ ord $\left.f_{j}\right)$ for $i \neq j$. Let $I=\left\{i \in\{1, \ldots, r\}:\right.$ ord $\left.f_{i}>1\right\}$. Then

$$
q_{0}(f)=\max _{i \in I}\left\{q_{0}\left(f_{i}\right)+\sum_{j \neq i} \text { ord } f_{j}\right\}=\max _{i \in I}\left\{q_{0}\left(f_{i}\right)-\text { ord } f_{i}\right\}+\text { ord } f
$$

if $I \neq \emptyset$ and $q_{0}(f)=$ ord $f$ if $I=\emptyset$.

Corollary $1.8([\mathrm{Li}])$. Suppose that $f=f_{1} \cdots f_{r}(r \geq 2)$ where $f_{i}$ are irreducible, pairwise transverse and ord $f_{i}>1$ for some $i \in\{1, \ldots, r\}$. Then

$$
\operatorname{Suff}_{0}(f)=\max _{i \in I}\left\{\left[q_{0}\left(f_{i}\right)\right]+\sum_{j \neq i} \text { ord } f_{j}\right\} \text {. }
$$

Proof. We apply the second part of Corollary 1.5 and Remark 1.7. In [Li] (p. 160) the above formula is given without assumptions imposed on $f_{i}$.

\section{Polar quotients and Puiseux series}

Let $\boldsymbol{C}\{X\}^{*}=\bigcup_{n \geq 1} \boldsymbol{C}\left\{X^{1 / n}\right\}$ be the ring of Puiseux series. If $f(X, Y) \in$ $C\{X, Y\}$ is a power series $Y$-regular of order $p>0$ i.e. such that ord $f(0, Y)=$ $p$, then $f(X, Y)=\prod_{i=1}^{p}\left(Y-y_{i}(X)\right) U(X, Y)$ where $y_{i}(X) \in C\{X\}^{*}$ are without constant term and $U(X, Y) \in \boldsymbol{C}\{X, Y\}$ is a unit i.e. $U(0,0) \neq 0$. We denote by Zer $f=\left\langle y_{1}(X), \ldots, y_{p}(X)\right\rangle$ the sequence $y_{1}(X), \ldots, y_{p}(X)$ regarded as unordered. To simplify the notation we write $y_{i}$ for $y_{i}(X)$. Let $f(X, Y) \in$ $C\{X, Y\}$ be a power series $Y$-regular of order $p>1$, then $(\partial f / \partial Y)(X, Y) \in$ $C\{X, Y\}$ is $Y$-regular of order $p-1>0$ and we can consider the roots of both sereis: Zer $f=\left\langle y_{1}, \ldots, y_{p}\right\rangle$ and $\operatorname{Zer}(\partial f / \partial Y)=\left\langle z_{1}, \ldots, z_{p-1}\right\rangle$. The following is basic for us

Lemma 2.1 (The Kuo-Lu lemma, [KL] Lemma 3.3). Suppose that $f \in$ $C\{X, Y\}$ has no multiple factors. Then for every $i, j \in\{1, \ldots, p\}, i \neq j$, there exists a $k \in\{1, \ldots, p-1\}$ such that 


$$
\left(^{*}\right) \operatorname{ord}\left(y_{i}-y_{j}\right)=\operatorname{ord}\left(y_{i}-z_{k}\right) .
$$

Moreover, for every $i \in\{1, \ldots, p\}$ and $k \in\{1, \ldots, p-1\}$ there exists a $j \in$ $\{1, \ldots, p\}$ such that $(*)$ holds.

A simple proof of the Kuo-Lu lemma without using perturbations of power series is given in [GP1], Lemma 3.2.

Proposition 2.2. With the notations and assumptions introduced above

$$
q_{0}(f, X)=\max _{i=1}^{p}\left\{\sum_{j \neq i} \operatorname{ord}\left(y_{j}-y_{i}\right)+\max _{j \neq i}\left\{\operatorname{ord}\left(y_{j}-y_{i}\right)\right\}\right\} .
$$

Proof. By definition

$$
q_{0}(f, X)=\sup \left\{\frac{(f, g)_{0}}{(X, g)_{0}}: g \text { is an irreducible factor of } \frac{\partial f}{\partial Y}\right\} .
$$

Using Zeuthen's rule for intersection multiplicity we get

$$
q_{0}(f, X)=\max _{k=1}^{p-1}\left\{\sum_{i=1}^{p} \operatorname{ord}\left(z_{k}-y_{i}\right)\right\} .
$$

Put $l_{i}=\sum_{j \neq i} \operatorname{ord}\left(y_{j}-y_{i}\right)+\max _{j \neq i}\left\{\operatorname{ord}\left(y_{j}-y_{i}\right)\right\}$ and choose $i_{0} \in\{1, \ldots, p\}$ such that $\max _{i=1}^{p}\left\{l_{i}\right\}=l_{i_{0}}$. Let $j_{0} \in\{1, \ldots, p\}$ be such that

$$
\max _{j \neq i_{0}}\left\{\operatorname{ord}\left(y_{j}-y_{i_{0}}\right)\right\}=\operatorname{ord}\left(y_{j_{0}}-y_{i_{0}}\right) .
$$

By the first part of the Kuo-Lu lemma there is a root $z=z_{k}$ of $\partial f / \partial Y=0$ such that

$$
\operatorname{ord}\left(z-y_{i_{0}}\right)=\operatorname{ord}\left(y_{j_{0}}-y_{i_{0}}\right)
$$

We will check that

$$
\operatorname{ord}\left(z-y_{i}\right) \geq \operatorname{ord}\left(y_{i_{0}}-y_{i}\right) \text { for all } i \neq i_{0} .
$$

Indeed, we have

$$
\operatorname{ord}\left(z-y_{i}\right) \geq \min \left\{\operatorname{ord}\left(z-y_{i_{0}}\right), \operatorname{ord}\left(y_{i_{0}}-y_{i}\right)\right\}=\operatorname{ord}\left(y_{i_{0}}-y_{i}\right)
$$

for

$$
\operatorname{ord}\left(z-y_{i_{0}}\right)=\operatorname{ord}\left(y_{j_{0}}-y_{i_{0}}\right)=\max _{j \neq i}\left\{\operatorname{ord}\left(y_{j}-y_{i_{0}}\right)\right\} \geq \operatorname{ord}\left(y_{i}-y_{i_{0}}\right)
$$

for a given $i \neq i_{0}$. We get 


$$
\begin{aligned}
\max _{i=1}^{p}\left\{l_{i}\right\} & =l_{i_{0}}=\sum_{j \neq i_{0}} \operatorname{ord}\left(y_{j}-y_{i_{0}}\right)+\operatorname{ord}\left(y_{j_{0}}-y_{i_{0}}\right) \\
& \leq \sum_{j \neq i_{0}} \operatorname{ord}\left(z-y_{j}\right)+\operatorname{ord}\left(z-y_{i_{0}}\right) \\
& =\sum_{j=1}^{p} \operatorname{ord}\left(z-y_{j}\right)
\end{aligned}
$$

by (4) and (3). Consequently $\max _{i=1}^{p}\left\{l_{i}\right\} \leq q_{0}(f, X)$ by (1). To check the inequality $q_{0}(f, X) \leq \max _{i=1}^{p}\left\{l_{i}\right\}$ let $z=z_{k}$ be a root of $\partial f / \partial Y=0$ such that

$$
q_{0}(f, X)=\sum_{i=1}^{p} \operatorname{ord}\left(z-y_{i}\right)
$$

Let $i_{1} \in\{1, \ldots, p\}$ be such that

$$
\max _{i=1}^{p}\left\{\operatorname{ord}\left(z-y_{i}\right)\right\}=\operatorname{ord}\left(z-y_{i_{1}}\right) .
$$

We will check that

$$
\operatorname{ord}\left(y_{i}-y_{i_{1}}\right) \geq \operatorname{ord}\left(y_{i}-z\right) \text { for } i \neq i_{1} .
$$

We have

$$
\begin{aligned}
\operatorname{ord}\left(y_{i}-y_{i_{1}}\right) & \geq \min \left\{\operatorname{ord}\left(y_{i}-z\right), \operatorname{ord}\left(y_{i_{1}}-z\right)\right\} \\
& =\min \left\{\operatorname{ord}\left(y_{i}-z\right), \max _{j=1}^{p}\left\{\operatorname{ord}\left(z-y_{j}\right)\right\}\right\} \\
& =\operatorname{ord}\left(y_{i}-z\right) .
\end{aligned}
$$

By the second part of the Kuo-Lu lemma there is a $j_{1} \in\{1, \ldots, p\}$ such that

$$
\operatorname{ord}\left(z-y_{i_{1}}\right)=\operatorname{ord}\left(y_{j_{1}}-y_{i_{1}}\right) \text {. }
$$

Therefore we get

$$
\begin{aligned}
q(f, X) & =\sum_{i=1}^{p} \operatorname{ord}\left(z-y_{i}\right)=\sum_{i \neq i_{1}} \operatorname{ord}\left(z-y_{i}\right)+\operatorname{ord}\left(z-y_{i_{1}}\right) \\
& \leq \sum_{i \neq i_{1}} \operatorname{ord}\left(y_{i}-y_{i_{1}}\right)+\operatorname{ord}\left(y_{j_{1}}-y_{i_{1}}\right) \\
& \leq \sum_{i \neq i_{1}} \operatorname{ord}\left(y_{i}-y_{i_{1}}\right)+\max _{i \neq i_{1}}\left\{\operatorname{ord}\left(y_{i}-y_{i_{1}}\right)\right\} \\
& =l_{i_{1}} \leq \max _{i=1}^{p}\left\{l_{i}\right\} .
\end{aligned}
$$

This ends the proof of (2.2). 


\section{Characteristic, order of contact and intersection multiplicity}

In this section we recall some notions of the theory of branches. Our main reference is $[\mathrm{Z}]$ (see also [D], [GP1], [GP2] for nontransverse case). Let $f=$ $f(X, Y) \in \boldsymbol{C}\{X, Y\}$ be an irreducible power series $Y$-regular of order $p>1$. Clearly $(f, X)_{0}=p$. The Propositions 3.1, 3.2 and 3.3 are well-known.

Proposition 3.1. Let Zer $f=\left\langle y_{1}, \ldots, y_{p}\right\rangle$. Put $b_{0}=p$. Then there exists a sequence of strictly positive integers $b_{1}<\cdots<b_{h}$ such that for every $i \in\{1, \ldots, p\}$ :

(i) $\left\{b \in \boldsymbol{R}: b=\operatorname{ord}\left(y_{i}-y_{j}\right)\right.$ for some $\left.j \neq i\right\}=\left\{b_{1} / b_{0}, \ldots, b_{h} / b_{0}\right\}$

(ii) $\sharp\left\{j \in\{1, \ldots, p\}\right.$ : ord $\left.\left(y_{i}-y_{j}\right)=b_{k} / b_{0}\right\}=B_{k-1}-B_{k}$ where $B_{k}=$ GCD. $\left(b_{0}, \ldots, b_{k}\right)$.

Proof. Let $i \in\{1, \ldots, p\}$. Then $y_{i}(X)=\eta\left(X^{1 / p}\right)$ where $\eta(T) \in C\{T\}$. The roots of $f(X, Y)=0$ form a cycle $\eta\left(w X^{1 / p}\right)$ where $w$ runs over the set $U(p)$ of $p$-th roots of unity. There exists strictly increasing sequence $b_{1}, \ldots, b_{h}$ such that $\operatorname{ord}\left(\eta\left(w X^{1 / p}\right)-\eta\left(X^{1 / p}\right)\right)=b_{k} / p$ if $w \in U\left(B_{k-1}\right) \backslash U\left(B_{k}\right)$ and $k=1, \ldots, p$. Clearly $\sharp\left(U\left(B_{k-1}\right) \backslash U\left(B_{k}\right)\right)=B_{k-1}-B_{k}$ and the proposition follows. For more details see [Z] (Chapter II) and [GP2] (Section 3).

We call $\left(b_{0}, b_{1}, \ldots, b_{h}\right)$ the characteristic sequence of $f$ (with respect to coordinates $X, Y)$. We put $\bar{b}_{k}=b_{k}+\left(1 / B_{k-1}\right) \sum_{i=1}^{k-1}\left(B_{i-1}-B_{i}\right) b_{i}$ for $k=1, \ldots$, $h$. The sum of an empty family is equal to zero. Thus we have $\bar{b}_{1}=b_{1}$. We put $\bar{b}_{0}=b_{0}$.

Proposition 3.2. The sequence $\bar{b}_{0}, \bar{b}_{1}, \ldots, \bar{b}_{h}$ is a system of generetors of the semigroup $\Gamma_{0}(f)$ with respect to $\bar{b}_{0}=(f, X)_{0}$ i.e. $\bar{b}_{k}=\min \left\{\Gamma_{0}(f) \backslash\left(N \bar{b}_{0}+\cdots+\right.\right.$ $\left.\left.\boldsymbol{N} \bar{b}_{k-1}\right)\right\}$ for $k=1, \ldots, h$ and $\boldsymbol{N} \bar{b}_{0}+\cdots+\boldsymbol{N} \bar{b}_{h}=\Gamma_{0}(f)$.

Proof. See $[\mathrm{Z}]$ (Théorème 3.9) and [GP2] (Proposition 3.2).

Let us consider two $Y$-regular power series $f, g \in C\{X, Y\}$. Let Zer $f=$ $\left\langle y_{1}(X), \ldots, y_{p}(X)\right\rangle$ and Zer $g=\left\langle z_{1}(X), \ldots, z_{q}(X)\right\rangle$. We define the order of contact of $f$ and $g$ (in coordinates $X, Y$ ) by putting

$$
\operatorname{cont}(f, g)=\max \left\{\operatorname{ord}\left(y_{i}(X)-z_{j}(X)\right): 1 \leq i \leq p, 1 \leq j \leq q\right\} .
$$

Clearly $\operatorname{cont}(f, g)=\operatorname{cont}(g, f)$. Moreover it is easy to check that $\operatorname{cont}(f, g)=$ $\max _{i=1}^{p}\left\{\operatorname{ord}\left(y_{i}(X)-z_{j}(X)\right)\right\}$ for every $j \in\{1, \ldots, q\}$.

Proposition 3.3. Suppose that ord $f(0, Y)>1$ and let $\left(b_{0}, \ldots, b_{h}\right)$ be the characteristic sequence of $f$. Let $k>0$ be the smallest integer such that $\operatorname{cont}(f, g)$ $\leq b_{k} / b_{0}$ (we put $\left.b_{h+1} / b_{0}=+\infty\right)$. Then

$$
\frac{(f, g)_{0}}{(X, g)_{0}}=\sum_{i=1}^{k-1}\left(B_{i-1}-B_{i}\right) \frac{b_{i}}{b_{0}}+B_{k-1} \operatorname{cont}(f, g) .
$$


Proof (see also [M], Proposition 2.4 where the case of generic coordinates is considered). We may assume that $\operatorname{cont}(f, g)=\operatorname{ord}\left(y_{1}-z_{1}\right)$. By Zeuthen's rule we get

$$
\frac{(f, g)_{0}}{(X, g)_{0}}=\sum_{i=1}^{p} \operatorname{ord}\left(y_{i}-z_{1}\right)
$$

Using Proposition 3.1 we check that

(a) the set $\left\{\operatorname{ord}\left(y_{1}-z_{1}\right), \ldots, \operatorname{ord}\left(y_{p}-z_{1}\right)\right\}$ is equal to the set $\left\{b_{1} / b_{0}, \ldots\right.$, $\left.b_{k-1} / b_{0}, \operatorname{cont}(f, g)\right\}$,

(b) $\sharp\left\{j \in\{1, \ldots, p\}: \operatorname{ord}\left(y_{j}-z_{1}\right)=b_{i} / b_{0}\right\}=B_{i-1}-B_{i}$,

(c) $\sharp\left\{j \in\{1, \ldots, p\}: \operatorname{ord}\left(y_{j}-z_{1}\right)=\operatorname{cont}(f, g)\right\}=B_{k-1}$

and Proposition 3.3 follows.

Using (3.1) and (3.3) we prove

Proposition 3.4. Let $f=f(X, Y) \in C\{X, Y\}$ be irreducible, Y-regular, ord $f(0, Y)>1$ of characteristic $\left(b_{0}, b_{1}, \ldots, b_{h}\right)$. Then

(i) $q_{0}(f, X)=\sum_{i=1}^{h}\left(B_{i-1}-B_{i}\right) b_{i} / b_{0}+b_{h} / b_{0}=B_{h-1} \bar{b}_{h} / \bar{b}_{0}$,

(ii) for every irreducible, Y-regular power series $g \in C\{X, Y\}$

$$
\frac{(f, g)_{0}}{(X, g)_{0}} \leq q_{0}(f, X) \text { if and only if } \operatorname{cont}(f, g) \leq \frac{b_{h}}{b_{0}}
$$

Proof. To prove (i) let us observe that by Proposition 3.1:

$$
\sum_{j \neq i} \operatorname{ord}\left(y_{i}-y_{j}\right)+\max _{j \neq i}\left\{\operatorname{ord}\left(y_{j}-y_{i}\right)\right\}=\sum_{i=1}^{h}\left(B_{i-1}-B_{i}\right) \frac{b_{i}}{b_{0}}+\frac{b_{h}}{b_{0}}=B_{h-1} \frac{\bar{b}_{h}}{\bar{b}_{0}}
$$

for every $i \in\{1, \ldots, p\}$. Then we use Proposition 2.2. To check (ii) let us suppose first that $\operatorname{cont}(f, g) \leq b_{h} / b_{0}$. Then the smallest $k>0$ such that $\operatorname{cont}(f, g) \leq b_{k} / b_{0}$ is less than or equal to $h$. By Proposition 3.3 we get

$$
\begin{aligned}
\frac{(f, g)_{0}}{(X, g)_{0}} & \leq \sum_{i=1}^{k-1}\left(B_{i-1}-B_{i}\right) \frac{b_{i}}{b_{0}}+B_{k-1} \frac{b_{k}}{b_{0}}=B_{k-1} \frac{\bar{b}_{k}}{\bar{b}_{0}} \\
& \leq \frac{B_{h-1} \bar{b}_{h}}{\bar{b}_{0}}=q_{0}(f, X)
\end{aligned}
$$

for the sequence $B_{k-1} \bar{b}_{k}$ is increasing for $k>0 \quad\left(\bar{b}_{k+1}-\left(B_{k-1} / B_{k}\right) \bar{b}_{k}=\right.$ $b_{k+1}-b_{k}>0$ for $\left.k=1, \ldots, h-1\right)$.

Now, suppose that $\operatorname{cont}(f, g)>b_{h} / b_{0}$. Again by Proposition 3.3 we get 


$$
\begin{aligned}
\frac{(f, g)_{0}}{(X, g)_{0}} & =\sum_{i=1}^{h}\left(B_{i-1}-B_{i}\right) \frac{b_{i}}{b_{0}}+\operatorname{cont}(f, g)>\sum_{i=1}^{h}\left(B_{i-1}-B_{i}\right) \frac{b_{i}}{b_{0}}+\frac{b_{h}}{b_{0}} \\
& =\frac{B_{h-1} \bar{b}_{h}}{\bar{b}_{0}}=q_{0}(f, X) .
\end{aligned}
$$

This ends the proof.

\section{Proof}

It is easy to check

LEMMA 4.1. If $\Phi$ is a local isomorphism i.e. a pair of power series without constant term such that $\mathrm{Jac} \Phi(0,0) \neq 0$ then

$$
q_{0}(f, t)=q_{0}(f \circ \Phi, t \circ \Phi) .
$$

Therefore to prove (1.1) and (1.3) it suffices to consider the case $t=X$.

Proof of Proposition 1.1. Let $\left(\bar{\beta}_{0}, \ldots, \bar{\beta}_{g}\right)$ be the minimal system of generators of $\Gamma_{0}(f)$. It is known $([\mathrm{D}], \mathrm{pp} .332-333)$ that three cases are possible

(a) $\bar{b}_{0}=\bar{\beta}_{0}$ then $h=g$ and $\left(\bar{b}_{0}, \ldots, \bar{b}_{g}\right)=\left(\bar{\beta}_{0}, \ldots, \bar{\beta}_{g}\right)$,

(b) $\bar{b}_{0}>\bar{\beta}_{0}$ is a multiple of $\bar{\beta}_{0}$, then $h=g+1$ and $\left(\bar{b}_{0}, \ldots, \bar{b}_{g+1}\right)=$ $\left(\bar{b}_{0}, \bar{\beta}_{0}, \ldots, \bar{\beta}_{g}\right)$

(c) $\bar{b}_{0}>\bar{\beta}_{0}$ and $\bar{b}_{0}$ is not a multiple of $\bar{\beta}_{0}$, then $h=g$ and $\left(\bar{b}_{0}, \bar{b}_{1}, \ldots, \bar{b}_{g}\right)=$ $\left(\bar{\beta}_{1}, \bar{\beta}_{0}, \bar{\beta}_{2}, \ldots, \bar{\beta}_{g}\right)$.

Therefore $B_{h-1} \bar{b}_{h}=\operatorname{GCD}\left(\bar{b}_{0}, \ldots, \bar{b}_{h-1}\right) \bar{b}_{h}=\operatorname{GCD}\left(\bar{\beta}_{0}, \ldots, \bar{\beta}_{g-1}\right) \bar{\beta}_{g}$ and Proposition 1.1 follows from (3.4)(i).

Proof of Theorem 1.3. It suffices to prove the following: if $f=f_{1} \cdots f_{r}$ $(r \geq 2)$ with $f_{i}$ irreducible, is $Y$-regular, then

$$
q_{0}(f, X)=\max _{i=1}^{r}\left\{\max \left\{q_{0}\left(f_{i}, X\right), \max _{j \neq i} \frac{\left(f_{i}, f_{j}\right)_{0}}{\left(X, f_{j}\right)_{0}}\right\}+\frac{1}{\left(X, f_{i}\right)_{0}} \sum_{j \neq i}\left(f_{i}, f_{j}\right)_{0}\right\} .
$$

According to Proposition 2.2 we have to calculate the quantities

$$
l_{i}=\sum_{j \neq i} \operatorname{ord}\left(y_{j}-y_{i}\right)+\max _{j \neq i}\left\{\operatorname{ord}\left(y_{j}-y_{i}\right)\right\} .
$$

Without restriction of the generality we may assume that $i=1$ and $y_{1}=y_{1}(X)$ is a root of the equation $f_{1}(X, Y)=0$. Let us suppose that ord $f_{1}(0, Y)>1$ and let $\left(b_{0}, \ldots, b_{h}\right)$ be the characteristic of $f_{1}$. Set

$$
I_{1}=\left\{i \neq 1: y_{i}=y_{i}(X) \text { is a root of } f_{1}(X, Y)=0\right\}
$$

and 
ON THE MAXIMAL POLAR QUOTIENT OF AN ANALYTIC PLANE CURVE

$$
I_{2}=\left\{i: y_{i}=y_{i}(X) \text { is a root of } f_{2}(X, Y) \cdots f_{r}(X, Y)=0\right\} .
$$

Therefore we can write

$$
\begin{aligned}
l_{1}= & \sum_{i \in I_{1}} \operatorname{ord}\left(y_{i}(X)-y_{1}(X)\right)+\sum_{i \in I_{2}} \operatorname{ord}\left(y_{i}(X)-y_{1}(X)\right) \\
& +\max \left\{\max _{i \in I_{1}}\left\{\operatorname{ord}\left(y_{1}(X)-y_{i}(X)\right)\right\}, \max _{i \in I_{2}}\left\{\operatorname{ord}\left(y_{i}(X)-y_{1}(X)\right)\right\}\right\} .
\end{aligned}
$$

We get

$$
\begin{gathered}
\sum_{i \in I_{1}} \operatorname{ord}\left(y_{i}(X)-y_{1}(X)\right)=\sum_{i=1}^{h}\left(B_{i-1}-B_{i}\right) \frac{b_{i}}{b_{0}} \text { by Proposition 3.1, } \\
\sum_{i \in I_{2}} \operatorname{ord}\left(y_{i}(X)-y_{1}(X)\right)=\frac{1}{\left(X, f_{1}\right)_{0}} \sum_{i \neq 1}\left(f_{1}, f_{i}\right)_{0} \text { by Zeuthen's rule, } \\
\max _{i \in I_{1}}\left\{\operatorname{ord}\left(y_{i}(X)-y_{1}(X)\right)\right\}=\frac{b_{h}}{b_{0}} \text { by Proposition 3.1, } \\
\max _{i \in I_{2}}\left\{\operatorname{ord}\left(y_{i}(X)-y_{1}(X)\right)\right\}=\max _{i \neq 1}\left\{\operatorname{cont}\left(f_{1}, f_{i}\right)\right\} \text { by definition. }
\end{gathered}
$$

Consequently we get

$$
\begin{aligned}
\left(^{*}\right) l_{1}= & \frac{1}{\left(X, f_{1}\right)_{0}} \sum_{i \neq 1}\left(f_{1}, f_{i}\right)_{0}+\sum_{i=1}^{h}\left(B_{i-1}-B_{i}\right) \frac{b_{i}}{b_{0}} \\
& +\max \left\{\frac{b_{h}}{b_{0}}, \max _{i \neq 1} \operatorname{cont}\left(f_{1}, f_{i}\right)\right\} .
\end{aligned}
$$

Let us consider two cases.

CASE 1. $\max _{i \neq 1}\left\{\operatorname{cont}\left(f_{1}, f_{i}\right)\right\} \leq b_{h} / b_{0}$. By Proposition 3.4 we have $q_{0}\left(f_{1}, X\right)=\sum_{i=1}^{h}\left(B_{i-1}-B_{i}\right) b_{i} / b_{0}+b_{h} / b_{0}$ and $\left(f_{1}, f_{i}\right)_{0} /\left(X, f_{i}\right)_{0} \leq q_{0}\left(f_{1}, X\right)$ for all $i \neq 1$. Therefore we get by $(*)$ :

$$
\begin{aligned}
l_{1} & =\frac{1}{\left(X, f_{1}\right)_{0}} \sum_{i \neq 1}\left(f_{1}, f_{i}\right)_{0}+\sum_{i=1}^{h}\left(B_{i-1}-B_{i}\right) \frac{b_{i}}{b_{0}}+\frac{b_{h}}{b_{0}} \\
& =\frac{1}{\left(X, f_{1}\right)_{0}} \sum_{i \neq 1}\left(f_{1}, f_{i}\right)+q_{0}\left(f_{1}, X\right) \\
& =\frac{1}{\left(X, f_{1}\right)_{0}} \sum_{i \neq 1}\left(f_{1}, f_{i}\right)_{0}+\max \left\{q_{0}\left(f_{1}, X\right), \max _{i \neq 1} \frac{\left(f_{1}, f_{i}\right)_{0}}{\left(X, f_{i}\right)_{0}}\right\} .
\end{aligned}
$$

CASE 2. $\max _{i \neq 1}\left\{\operatorname{cont}\left(f_{1}, f_{i}\right)\right\}>b_{h} / b_{0}$. Let us fix $i \neq 1$. If $\operatorname{cont}\left(f_{1}, f_{i}\right)>$ $b_{h} / b_{0}$ then 


$$
\frac{\left(f_{1}, f_{i}\right)_{0}}{\left(X, f_{i}\right)_{0}}=\sum_{i=1}^{h}\left(B_{i-1}-B_{i}\right) \frac{b_{i}}{b_{0}}+\operatorname{cont}\left(f_{1}, f_{i}\right)
$$

by (3.3), if $\operatorname{cont}\left(f_{1}, f_{i}\right) \leq b_{h} / b_{0}$ then

$$
\frac{\left(f_{1}, f_{i}\right)_{0}}{\left(X, f_{i}\right)_{0}} \leq \sum_{i=1}^{h}\left(B_{i-1}-B_{i}\right) \frac{b_{i}}{b_{0}}
$$

again by (3.3). Therefore

$$
\max _{i \neq 1} \frac{\left(f_{1}, f_{i}\right)_{0}}{\left(X, f_{i}\right)_{0}}=\sum_{i=1}^{h}\left(B_{i-1}-B_{i}\right) \frac{b_{i}}{b_{0}}+\max _{i \neq 1}\left\{\operatorname{cont}\left(f_{1}, f_{i}\right)\right\} .
$$

Using $(*)$ we get

$$
\begin{aligned}
l_{1}= & \frac{1}{\left(X, f_{1}\right)_{0}} \sum_{i \neq 1}\left(f_{1}, f_{i}\right)_{0}+\sum_{i=1}^{h}\left(B_{i-1}-B_{i}\right) \frac{b_{i}}{b_{0}}+\max _{i \neq 1}\left\{\operatorname{cont}\left(f_{1}, f_{i}\right)\right\} \\
= & \frac{1}{\left(X, f_{1}\right)_{0}} \sum_{i \neq 1}\left(f_{1}, f_{i}\right)_{0}+\max _{i \neq 1} \frac{\left(f_{1}, f_{i}\right)_{0}}{\left(X, f_{i}\right)_{0}} \\
= & \frac{1}{\left(X, f_{1}\right)_{0}} \sum_{i \neq 1}\left(f_{1}, f_{i}\right)_{0}+\max \left\{q_{0}\left(f_{1}, X\right), \max _{i \neq 1} \frac{\left(f_{1}, f_{i}\right)_{0}}{\left(X, f_{i}\right)_{0}}\right\} \\
& \text { for } q_{0}\left(f_{1}, X\right)=\sum_{i=1}^{h}\left(B_{i-1}-B_{i}\right) \frac{b_{i}}{b_{0}}+\frac{b_{h}}{b_{0}}<\max _{i \neq 1} \frac{\left(f_{1}, f_{i}\right)_{0}}{\left(X, f_{i}\right)_{0}} .
\end{aligned}
$$

It remains to consider the case ord $f_{1}(0, Y)=1$. Then $\left(X, f_{1}\right)_{0}=1, q_{0}\left(f_{1}, X\right)=$ $-\infty$ and we get easily

$$
l_{1}=\sum_{j \neq 1}\left(f_{1}, f_{j}\right)_{0}+\max _{j \neq 1} \frac{\left(f_{1}, f_{j}\right)_{0}}{\left(X, f_{j}\right)_{0}} .
$$

This ends the proof.

\section{Appendix}

We reprove here the main result of $[\mathrm{KL}]$.

Let $f=f(X, Y) \in C\{X, Y\}$ be a power series $Y$-regular of order $p=\operatorname{ord} f$. Let $y_{i}=y_{i}(X) \in C\{X\}^{*}(i=1, \ldots, p)$ be the sequence of all solutions (without constant term) of the equation $f(X, Y)=0$. We put

$$
l_{i}=\sum_{j \neq i} \operatorname{ord}\left(y_{i}-y_{j}\right)+\max _{j \neq i}\left\{\operatorname{ord}\left(y_{i}-y_{j}\right)\right\} \quad \text { for } i=1, \ldots, p .
$$

Then we have 
ON THE MAXIMAL POLAR QUOTIENT OF AN ANALYTIC PLANE CURVE

Kuo and Lu's formula for the Lojasiewicz exponent ([KL], Theorem A). With the above notation assume that $f$ is a reduced power series with ord $f>1$. Then, we have

$$
\mathscr{L}_{0}(f)=\max _{i=1}^{p}\left\{l_{i}-1\right\}
$$

Proof. By Proposition 2.2 we have $q_{0}(f, X)=\max _{i=1}^{p}\left\{l_{i}\right\}$. On the other hand $q_{0}(f, X)=q_{0}(f)$ by the second part of Corollary 1.4. Then we use the first part of Corollary 1.5.

Note here that the original result was proved for distinguished polynomials. The quantities $l_{i}$ was defined in Kuo and Lu's work by means of some perturbations of the roots $y_{i}=y_{i}(X)$. The simple expressions for $l_{i}$ we use are due to Bogusławska ([B], Lemma 3).

Let $c_{i}=\max _{j \neq i}\left\{\operatorname{ord}\left(y_{i}-y_{j}\right)\right\}$ for $i=1, \ldots, p$. According to [KL] a root $y_{i}$ is minimal if for any root $y_{j}$ with $\operatorname{ord}\left(y_{i}-y_{j}\right)=c_{i}$ we get $c_{j} \leq c_{i}$. If is easy to check that if $c_{i}<c_{k}$ then $l_{i}<l_{k}$. Hence we get

Corollary to Kuo and Lu's formula $\left([\mathrm{KL}]\right.$, Theorem $\left.\mathrm{A}^{\prime}\right)$. We have

$$
\mathscr{L}_{0}(f)=\max _{i \in I}\left\{l_{i}-1\right\} .
$$

where $I$ is the set of all $i \in\{1, \ldots, p\}$ such that the root $y_{i}$ is minimal.

For every branch $f$ we denote by $K_{0}(f)$ the knot corresponding to $f$. Let $f$ be a branch of characteristic $\left(\beta_{0}, \ldots, \beta_{q}\right)$. We define the self-link $\sigma_{0}(f)$ of $f$ to be $\sigma_{0}(f)=\operatorname{link}\left(K_{0}(f), K_{0}(\tilde{f})\right)$ where $\tilde{f}$ is a branch of characteristic $\left(\beta_{0}, \ldots, \beta_{q}\right)$ such that $\operatorname{cont}(f, \tilde{f})=\beta_{g} / \beta_{0}$ (see $[\mathrm{KL}]$, pp. 300-301). Then $\sigma_{0}(f)=(f, \tilde{f})_{0}=$ $\operatorname{GCD}\left(\bar{\beta}_{0}, \ldots, \bar{\beta}_{g-1}\right) \bar{\beta}_{g}=q_{0}(f)$ (ord $\left.f\right)$ by Proposition 3.3.

In $[\mathrm{KL}]$ the authors assert ([KL], Corollary to Theorem $\mathrm{B})$ the following: "suppose that $y_{1}=y_{1}(X)$ is a minimal root of $f=f_{1} \cdots f_{r}$ and let $f_{i}=f_{i}(X, Y)$ be such that $f_{i}\left(X, y_{1}(X)\right)=0$. Assume that knots $K_{0}\left(f_{i}\right)$ and $K_{0}\left(f_{j}\right)$ are not isotopic for $i \neq j$. Then

$$
\left(\text { ord } f_{i}\right) l_{1}=\sum_{j \neq i}\left(f_{i}, f_{j}\right)_{0}+\sigma_{0}\left(f_{i}\right) . "
$$

The example given below shows that the above statement (and consequently Theorem $\mathrm{B}$ of $[\mathrm{KL}]$ ) are not true.

Example. Let $1<\beta_{0}<\cdots<\beta_{g}, g>1$ be a sequence of coprime integers such that the divisors $\operatorname{GCD}\left(\beta_{0}, \ldots, \beta_{i}\right)$ form a strictly decreasing sequence. Let $f_{1}(X, Y)=0$ (resp. $f_{2}(X, Y)=0$ ) be the minimal equation of the Puiseux series $y_{1}=X^{\beta_{1} / \beta_{0}}+\cdots+X^{\beta_{g-1} / \beta_{0}} \quad$ (resp. $\left.\quad y_{2}=X^{\beta_{1} / \beta_{0}}+\cdots+X^{\beta_{g} / \beta_{0}}\right)$. Let $f=f_{1} f_{2}$. The series $f_{1}$ and $f_{2}$ have different characteristics and hence the knots $K_{0}\left(f_{1}\right)$ and $K_{0}\left(f_{2}\right)$ are not isotopic. For every root $y_{i}$ of $f$ we get $c_{i}=\operatorname{cont}\left(f_{1}, f_{2}\right)=\beta_{g} / \beta_{0}$, 
therefore all roots of $f$ are minimal. The calculation from the proof of Theorem 1.3 (Section 4 of this paper, Case 2) gives

$$
l_{1}=\frac{\left(f_{1}, f_{2}\right)_{0}}{\operatorname{ord} f_{1}}+\frac{\left(f_{1}, f_{2}\right)_{0}}{\operatorname{ord} f_{2}}
$$

and

$$
q_{0}\left(f_{1}\right)<\frac{\left(f_{1}, f_{2}\right)_{0}}{\operatorname{ord} f_{2}}
$$

Therefore

$$
\begin{aligned}
\left(\operatorname{ord} f_{1}\right) l_{1} & =\left(f_{1}, f_{2}\right)_{0}+\operatorname{ord} f_{1} \frac{\left(f_{1}, f_{2}\right)_{0}}{\operatorname{ord} f_{2}} \\
& >\left(f_{1}, f_{2}\right)_{0}+\left(\operatorname{ord} f_{1}\right) q_{0}\left(f_{1}\right)=\left(f_{1}, f_{2}\right)_{0}+\sigma_{0}\left(f_{1}\right) .
\end{aligned}
$$

This contradicts the statement quoted above.

\section{REFERENCES}

[B] M. Boguslawska, On the Lojasiewicz exponent of the gradient of holomorphic functions, Bull. Polish Acad. Sci. Math., 47 (1999), 337-343.

[CA] E. Casas-Alvero, Singularities of Plane Curves, Cambridge Univ. Press, to appear.

[D] F. Delgado de la Mata, A factorization theorem for the polar of a curve with two branches, Compositio Math., 92 (1994), 327-375.

[E] H. EgGers, Polarinvarianten und die Topologie von Kurvensingularitaen, Bonner Math. Schriften 147, 1983.

[Eph] R. Ephraim, Special polars and curves with one place at infinity, Singularities, Proc. Symp. Pure Math. vol. 40, Part I, AMS, 1983, 353-359.

[Ga] E. García-Barroso, Sur les courbes polaires d'une courbes plane réduite, Proc. London Math. Soc., 81 (2000), 1-28.

[GP1] J. Gwoździewicz, A. PŁoski, On the Merle formula for polar invariants, Bull. Soc. Sci. Lett. Łódź, 41 (1991), 61-67.

[GP2] J. Gwoździewicz, A. Ploski, On the approximate roots of polynomials, Ann. Polon. Math., 60 (1995), 199-210.

[KL] T. C. Kuo AND Y. C. LU, On analytic function germ of two complex variables, Topology, 16 (1977), 299-310.

[KP] T. C. KuO AND A. PARusiński, Newton polygon relative to an arc (J. W. Bruce and F. Tari eds.), Real and Complex Singularities, Chapman \& Hall/CRC, 1998, 76-93.

[Lê] LÊ VAN THÀNH, Affine polar quotients and singularity at infinity of an algebraic plane curve, Singularity Theory, 19 August-6 September 1991 (D. T. Lê, K. Saito, B. Teissier eds.), Word Scientific, 336-344.

[Li] B. Lichtin, Estimates and formulae for the $C^{0}$-degree of sufficiency of plane curves, Singularities, Proc. Symp. Pure Math., vol. 40, Part II, AMS, 1983, 155-160.

[LMW] Lê Dung Trang, F. Michel, C. Weber, Courbes polaires et topologie des courbes planes, Ann. Scient. École Norm. Sup. (4), 24 (1991), 141-169.

[LW] LÊ Dung Trang, C. Weber, Équisingularité dans les pinceaux de germes de courbes planes et $C^{0}$-suffisance, l'Enseign. Math., 43 (1997), 355-380. 
[M] M. Merle, Invariants polaires des courbes planes, Invent. Math., 41 (1977), 103-111.

[T] B. Teissier, Variétés polaires, Invent. Math., 40 (1977), 267-292.

[Z] O. ZARISKI, Le Problème des Modules pour les Branches Planes, Hermann, 1986.

DePartMent of MATHEMATics

TECHNICAL UNIVERSITY

AL. 1000 L PP 7

25-314 Kielce, Poland

e-mail: matap@tu.kielce.pl 